\title{
On Subspace Identification of Cascade Structured Systems
}

\author{
Per Hägg, Bo Wahlberg and Henrik Sandberg
}

\begin{abstract}
In identification it is important to take a priori structural information into account in many applications, something that is difficult when using subspace methods. Here will study how to incorporate a special structure, a cascade structure with two subsystems. Two new methods are derived for estimating system with this structure. The problem when using subspace identification on cascade structured system is that the states from the first subsystem are mixed with states from the second subsystem via a unknown similarity transform. The first indirect method finds a similarity transform that takes the system back to a form such that the subsystems can be recovered. The second method uses the fact that the structure of the extended observability matrix is known for cascade systems. However, it only works when both subsystems have order one. In practice this is still a common case. The results of the two methods seem promising, as illustrated by applying the methods to a real process, the double tank process. The performance is comparable with state of the art methods. Finally the problem of optimal input design for cascade systems are introduced, and illustrated by a simple example.
\end{abstract}

\section{INTRODUCTION}

System identification concerns the problem of construction and validation of mathematical models of dynamical systems from experimental data. Most of the classical identification methods deal with Single-Input Single-Output (SISO) systems. Many of these methods can, however, be generalized to Multi-Input Multi-Output (MIMO) systems. An identification technique called subspace identification has proven useful when dealing with MIMO systems [1], [2]. This is a blackbox technique for identification of state space models. It is, however, important in identification to take a priori information about the structure of the system into account. This has proven difficult when using subspace methods as well as for other methods.

The objective of this contribution is to study a few methods to identify systems with a cascade, or serial, structure as illustrated in Fig. 1, using subspace methods. The current work has been motivated by a discussion on use of subspace identification in process industry presented in [3].

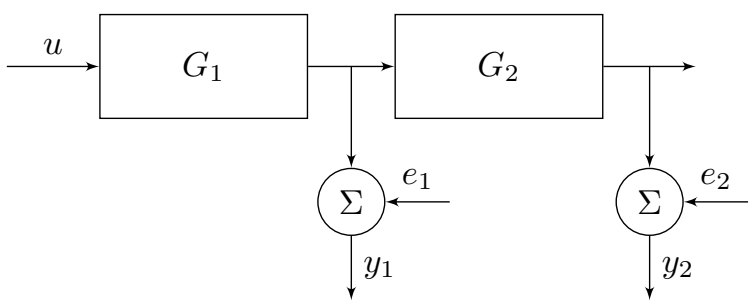

Fig. 1. Cascade system.

In particular systems with one input signal and two output

This work was partially supported by the Swedish Foundation for Strategic Research, the Swedish Research Council, the Linnaeus Center ACCESS at KTH and the European Commission through the WIDE project.

The authors are with the Automatic Control Lab and ACCESS, School of Electrical Engineering, KTH, SE-100 44 Stockholm, Sweden. E-mail: \{pehagg, bo, hsan\}@kth.se signals will be studied. The input-output relations for this system are

$$
\begin{aligned}
& y_{1}(t)=G_{1}(q) u(t)+e_{1}(t) \\
& y_{2}(t)=G_{1}(q) G_{2}(q) u(t)+e_{2}(t)
\end{aligned}
$$

The input signal is denoted $u(t)$ and the outputs, $y_{1}(t)$ and $y_{2}(t)$ respectively. The signals $e_{1}(t)$ and $e_{2}(t)$ are assumed zero mean white Gaussian measurement noise processes with variances $\lambda_{1}$ and $\lambda_{2}$. The transfer functions $G_{1}(q)$ and $G_{2}(q)$ are assumed stable. Here $q$ denotes the standard shift operator, i.e. $q^{-1} u(t)=u(t-1)$. The notation $G_{3}(q)=$ $G_{2}(q) G_{1}(q)$ will be used throughout this paper.

The problem is hence to identify the subsystems $G_{1}(q)$ and $G_{2}(q)$ from data $\left\{u(t), y_{1}(t), y_{2}(t)\right\}, t=1 \ldots N$. Any single-input multiple output method could be used, but it is often not straightforward to impose the cascade model structure.

The contribution of this paper is two new methods that integrate structure into the subspace identification of cascade structured systems. The methods are applied to a real system, the double tank process. The method shows comparable performance with state of the art methods. It is hard to say something about the statistical properties for these methods, however these estimates could be used as initial estimates for a Prediction Error Method (PEM) or the Maximum Likelihood (ML) method.

The problem of optimal input design for cascade systems is also introduced.

The outline of this paper is as follows. First an overview of other methods of identifying cascade systems is given in Section II. In Section III the problem with subspace identification of cascade systems are presented and some properties of cascade systems necessary for the new methods are studied. Section IV introduces two methods for identifying cascade structured systems using subspace methods. These methods are applied to a real process, the double tank system, in Section V. The problem of designing an optimal input for identification of cascade systems are studied in Section VI. Finally, Section VII concludes the paper.

\section{Other Methods And Related Work}

One direct approach to identify the subsystems would be to first identify $G_{1}(q)$ from data $\left\{u(t), y_{1}(t)\right\}$ and then in a second step identify $G_{2}(q)$ from data $\left\{\hat{u}_{2}(t), y_{2}(t)\right\}$, where $\hat{u}_{2}$ is an estimate of the input to the second subsystem $G_{2}(q)$. If the model estimate $\hat{G}_{1}(q)$ is good, one could use $\hat{u}_{2}=\hat{G}_{1}(q) u(t)$. If the noise variance is low for the first measurement noise process one could use $\hat{u}_{2}(t)=y_{1}(t)$. It is also possible to use an optimal predictor of $u_{2}(t)$ based on the statistical properties of $e_{1}(t)$.

It is also possible to apply a Prediction Error Method (PEM) or the Maximum Likelihood (ML) method to this 
problem [1]. But because of the product $G_{1}(q) G_{2}(q)$, simple linear model structures such as OE or ARX are not directly applicable. It can however be shown that structured PEM and structured ML are statistically optimal methods for this problem. The statistical properties of these identified models are analyzed in [4], [5]. The Prediction Error based methods or Maximum Likelihood methods often involve solving non-convex optimization problem. It can therefore be difficult to guarantee that during the optimization the global optimum will be found. A way to solve this is to try to find good initial estimates, hopefully lying in the region of attraction for the global optimum. The two proposed methods in this paper could be a way to find such initial estimates.

The problem of imposing some structure into the subspace method could be found in [6] where they try to identify OE and ARMAX models using subspace methods. In [7] and [8] they want to guarantee that the identified model with a subspace method is stable when the true linear system is known to be stable.

Another approach proposed in [9] is to identify the transfer functions $G_{1}$ and $G_{3}=G_{1} G_{2}$ and then obtain an estimate of $G_{2}$ by solving the following standard $H_{\infty}$-model matching problem

$$
\hat{G}_{2}=\arg \inf _{Q \in R H_{\infty}}\left\|\hat{G}_{3}-Q \hat{G}_{1}\right\|_{\infty}
$$

where $\hat{G}_{1}$ and $\hat{G}_{3}$ are the identified models of $G_{1}$ and $G_{3}$ respectively. Typically the order of $\hat{G}_{2}$ is equal to the order of $\hat{G}_{1}$ plus the order of $\hat{G}_{3}$. The order of $G_{2}$ should be the order of $G_{3}$ minus the order of $G_{1}$ if there is no pole-zero cancelation. In [9] they solve this by using structured model reduction to reduce the order of $G_{2}$.

\section{Subspace Identification of Cascade Systems}

In this section some basic properties for cascade systems that are needed for the new identification methods, are presented. The two new methods will be based on subspace identification. A good overview of the algorithm can be found in [2] and [1].

One natural realization of the system (1) on state space form is

$$
\begin{aligned}
{\left[\begin{array}{c}
x_{2}(t+1) \\
x_{1}(t+1)
\end{array}\right] } & =\left[\begin{array}{cc}
A_{2} & B_{2} C_{1} \\
0 & A_{1}
\end{array}\right]\left[\begin{array}{l}
x_{2}(t) \\
x_{1}(t)
\end{array}\right]+\left[\begin{array}{c}
0 \\
B_{1}
\end{array}\right] u(t) \\
{\left[\begin{array}{l}
y_{2}(t) \\
y_{1}(t)
\end{array}\right] } & =\left[\begin{array}{cc}
C_{2} & 0 \\
0 & C_{1}
\end{array}\right]\left[\begin{array}{l}
x_{2}(t) \\
x_{1}(t)
\end{array}\right]+\left[\begin{array}{l}
e_{2}(t) \\
e_{1}(t)
\end{array}\right]
\end{aligned}
$$

where $x_{1}(t) \in \mathbb{R}^{n_{1}}, x_{2}(t) \in \mathbb{R}^{n_{2}}$ and,

$$
G_{1}(q)=C_{1}\left(q I-A_{1}\right)^{-1} B_{1}, \quad G_{2}(q)=C_{2}\left(q I-A_{2}\right)^{-1} B_{2} .
$$

This special structure of the state space matrices, where the states $x_{1}(t)$ correspond to the first subsystem and the states $x_{2}(t)$ correspond to the second subsystem, will be called a realization in cascade form. Note that the matrix $B_{2} C_{1}$ should have $\operatorname{rank}\left(B_{2} C_{1}\right)=1$.

Just applying a standard subspace method to the system
(1) would return an estimate in the form

$$
\begin{aligned}
x(t+1) & =A x(t)+B u(t) \\
y_{1}(t) & =C_{1} x(t) \\
y_{2}(t) & =C_{2} x(t)
\end{aligned}
$$

A state space realization is not unique with respect to the input-output relation, i.e. the system is only identified up to an unknown similarity transform. In general the states from the first subsystem will be mixed with the states from the second subsystem, due to this unknown transform. The first and second subsystems could hence not be directly separated. If the similarity transform somehow was known, the system could be transformed back to cascade form (3) and the state space matrices for $G_{1}$ and $G_{2}$ could easily be recovered. In the first proposed method a transform that transforms the system back to cascade form is found and from this the subsystems are recovered.

Basically, the subspace method forms an estimate of the extended observability matrix from input-output data. As discussed before the estimate is a transformed estimate of the true observability matrix. The estimated extended observability matrix, $\tilde{O}_{r}$, has the form [1]

$$
\tilde{O}_{r}=\left[\begin{array}{c}
C \\
C A \\
\vdots \\
C A^{r-1}
\end{array}\right] \tilde{T}+\tilde{E}_{N}
$$

where $\tilde{T}$ is an unknown transformation of full rank and $\tilde{E}_{N}$ is an unknown matrix due to noise. The model order is determined by studying the singular values for the extended observability matrix and keep the $n$ most significant values. When the model order has been selected the estimate of $\hat{C}$ and $\hat{A}$ can be calculated from the extended observability matrix by solving a linear least square problem. The matrices $\hat{B}$ and $\hat{D}$ can then be found by solving a linear regression problem. [2], [1].

For the system on cascade form (3) the extended observability matrix becomes

$$
\begin{aligned}
& \tilde{O}_{r} \tilde{T}=\left[\begin{array}{c}
C \\
C A \\
\vdots \\
C A^{r-1}
\end{array}\right]\left[\begin{array}{cc}
\tilde{T}_{11} & \tilde{T}_{12} \\
\tilde{T}_{21} & \tilde{T}_{22}
\end{array}\right]= \\
& {\left[\begin{array}{cc}
C_{1} \tilde{T}_{11} & C_{1} \tilde{T}_{12} \\
C_{2} \tilde{T}_{21} & C_{2} \tilde{T}_{22} \\
C_{1} A_{1} \tilde{T}_{11} & C_{1} A_{1} \tilde{T}_{12} \\
\star & C_{2} B_{2} C_{1} T_{12}+C_{2} A_{2} \tilde{T}_{22} \\
C_{1} A_{1}^{2} \tilde{T}_{11} & C_{1} A_{1}^{2} \tilde{T}_{12} \\
\star & \left(C_{2} B_{2} C_{1} A_{1}+C_{2} A_{2} B_{2} C_{1}\right) \tilde{T}_{12}+C_{2} A_{2}^{2} \tilde{T}_{22} \\
\vdots & \vdots
\end{array}\right]}
\end{aligned}
$$

Some repeated values are replaced by stars $(\star)$ due to space limitations. This fundamental structure of the extended observability matrix will be used to derive the second method to identify the subsystems.

\section{IDENTIFICATION METHODS}

The observations made in the previous section are used to formulate two methods for identification of cascade systems. 


\section{A. Method 1: Indirect Method}

The main idea of this method is to find a similarity transform for the identified system $G_{3}$ such that the transformed system is in cascade form (3).

This method of finding a similarity transform that brings the system to cascade form was proposed in [10]. The transformation matrix is parameterized and a set of equations that has to be solved to get the system to cascade form is formulated. It is shown that the number of parameters is less than the number of equations needed to be solved. Due to uncertainties this problem does not in general have an exact solution. In [10] no method of solving this problem is presented. The proposed method here solves this problem by finding a similarity transform that takes the system to cascade form while minimizing the mean square error between the estimated output and the measured output. The method introduced here is related to the method proposed in [6] where OE and ARMAX models are estimated with subspace methods by finding suitable transformations, to get the system to the desired form. The method denoted method 1 has three steps:

Step 1: Identify the state space matrices $\hat{A}_{1}, \hat{C}_{1}, \hat{B}_{1}$ from data $\left\{u(t), y_{1}(t)\right\}, t=1 \ldots N$ with order $n_{1}$ and $\hat{A}_{3}, \hat{C}_{3}$ and $\hat{B}_{3}$ using $\left\{u(t), y_{2}(t)\right\}, t=1 \ldots N$ with order $n_{3}$. The order of the second subsystem is calculated as $n_{2}=n_{3}-n_{1}$. Step 2: Find a transformation, $T$ by solving the following optimization problem,

$$
\begin{aligned}
& \min _{T, \tilde{B}_{1}} \frac{1}{\lambda_{1}} \sum_{t=1}^{N}\left(\hat{C}_{1}\left(q I-\hat{A}_{1}\right)^{-1} \hat{B}_{1} u(t)-y_{1}(t)\right)^{2}+ \\
& \frac{1}{\lambda_{2}} \sum_{t=1}^{N}\left(\hat{C}_{3} T\left(q I-T^{-1} \hat{A}_{3} T\right)^{-1}\left(\begin{array}{c}
0 \\
\tilde{B}_{1}
\end{array}\right) u(t)-y_{2}(t)\right)^{2} \\
& \text { s.t } T^{-1} \hat{A}_{3} T \text { and } \hat{C}_{3} T \text { are in cascade form. }
\end{aligned}
$$

where $\lambda_{1}$ and $\lambda_{2}$ are the measurement noise variances or can be seen as some weighting if the variances are unknown.

The $D$-matrices are assumed zero here but an extension to non-zero $D$-matrices should be easy. Some of the constraints should be chosen such that the lower right corner of $T^{-1} \hat{A}_{3} T$ has approximately the same dynamics as the identified system $\hat{G}_{1}$ and that the lower left corner should contain zeros. The upper right corner of $T^{-1} \hat{A}_{3} T$ corresponding to $B_{2} C_{1}$ should have rank 1 . It is not obvious how these constraints should be chosen when the first subsystem has a order larger than one. Hence the optimization problem stated above is in general hard to solve.

Let us study the constraints in more detail. First look at the constraint that $\hat{C}_{3} T$ should be on cascade form. This means that the last $n_{1}$ elements of $\hat{C}_{3} T$ should be equal to zero. This in turns means that the last $n_{1}$ columns of $T$ must be in the kernel space of $\hat{C}_{3}$. The second set of constraints is that $T^{-1} \hat{A}_{3} T$ should be on cascade form. This means that the lower right corner should have similar dynamics as the first identified subsystem $\hat{G}_{1}$ and that the lower left corner should be the zero matrix.

If we use Schur factorization[11] on $\hat{A}_{3}$

$$
\hat{A}_{3}=U \tilde{A}_{3} U^{*}
$$

then $\tilde{A}_{3}$ is similar to $\hat{A}_{3}$ and has the lower left corner equal to the zero matrix. If the Schur factorization is performed such that the eigenvalues closest to the eigenvalues of the first subsystem is located in the lower right corner of $\tilde{A}_{3}$, the first $n_{2}$ columns of $T$ should be chosen as the first columns of $U$. This is summarized in a proposition.

Proposition 1: If the last $n_{1}$ columns in $T$ spans the kernel space of $C_{3}$ and the first $n_{2}$ columns span the space corresponding to the $n_{2}$ columns of $U$, where $U$ is given from the Schur factorization of $A_{3}=U \tilde{A}_{3} U^{*}$ such that the dynamics from the first system is in the lower right corner of the block-triangular matrix $\tilde{A}_{3}$. The system transformed with $T$ will be on cascade form.

Using Proposition 1, the optimization problem (5) could be simplified. If the optimization is instead performed over linear combinations of the vectors spanning the kernel space of $C_{3}$ and over scaled versions of the appropriate columns of $U$ from the Schur factorization. Denoting the order of the kernel space by $n_{K}=n_{2}+n_{1}-1$ gives

$$
T=\left[k_{1} u_{1} \ldots k_{n_{2}} u_{n_{2}}, \sum_{i=1}^{n_{K}} k_{i+n_{2}} c_{i} \ldots \sum_{i=1}^{n_{K}} k_{i+n_{2}+\left(n_{1}-1\right) n_{K}} c_{i}\right]
$$

where $u_{i}$ is the $i$ :th column of $\mathrm{U}$ from the Schur factorization (6) and $c_{i} \in \operatorname{Ker}\left(\hat{C}_{3}\right)$. The optimization is performed over $k_{i}$ $i=1 \ldots\left(n_{2}+n_{1}\left(n_{2}+n_{1}-1\right)\right)$. The number of optimization parameters are reduced from $\left(n_{1}+n_{2}\right)^{2}$.

Finally, the constraint that the rank of the upper right corner of $T^{-1} \hat{A}_{3} T$, denoted $\hat{A}_{12}$, should be one is hard to incorporate. Instead some heuristics could be used. Here we will use that the upper right corner should equal $B_{2} C_{1}$. Denote the estimate of the system matrix of the first subsystem from $T^{-1} \hat{A}_{3} T$ as $\bar{A}_{1}$. We denote $\bar{C}_{1}(T)$ as the transformed matrix of $\hat{C}_{1}$ where the transform is a similarity transform between the identified $\hat{A}_{1}$ and $\bar{A}_{1}$. Ideally the eigenvalues of $\hat{A}_{1}$ should be the same as the eigenvalues $\bar{A}_{1}$. If this is the case a similarity transform could easily be found. In general this is not the case. One solution to this is to transform both $\hat{A}_{1}$ and $\bar{A}_{1}$ to upper triangular form, then replace the eigenvalues in the diagonal of $\bar{A}_{1}$ by the corresponding in $\hat{A}_{1}$. This way the two matrices are similar and a similarity transform could be found. An estimate of $B_{2}$ is then given by

$$
\hat{B}_{2}=\hat{A}_{12} \bar{C}_{1}(T)^{\dagger}
$$

where $(\dagger)$ denotes the pseudo inverse. We now formulate the following optimization problem

$$
\begin{aligned}
& \min _{T, \tilde{B}_{1}} \frac{1}{\lambda_{1}} \sum_{t=1}^{N}\left(\hat{C}_{1}\left(q I-\hat{A}_{1} T\right)^{-1} \tilde{B}_{1} u(t)-y_{1}(t)\right)^{2}+ \\
& \frac{1}{\lambda_{2}} \sum_{t=1}^{N}\left(\hat{C}_{2}\left(q I-\hat{A}_{2}\right)^{-1} \hat{B}_{2} \hat{C}_{1}\left(q I-\hat{A}_{1} T\right)^{-1} u(t)-y_{2}(t)\right)^{2}
\end{aligned}
$$

where

$$
\begin{aligned}
& \hat{C}_{2}=(C T)_{1: n_{2}} \\
& \hat{B}_{2}=\hat{A}_{12} \bar{C}_{1}^{\dagger} \\
& \hat{A}_{2}=\left(T^{-1} \hat{A}_{3} T\right)_{1: n_{2}, 1: n_{2}} .
\end{aligned}
$$


The optimization is performed over columns of $T$ defined in (7) and $\tilde{B}_{1}$. This heuristics solves the problem with the rank condition in many cases.

Step 3: When the transformation is found the system is transformed to cascade form. From this the estimates of the state space matrices for the second subsystem $\hat{A}_{2}$ and $\hat{C}_{2}$ can easily be recovered. The matrix estimate $\hat{B}_{2}$ could be calculated from the matrix product $B_{2} C_{1}$ as described above.

\section{B. Method 2: Direct Method}

The second method uses the fact that the structure of the extended observability matrix is known for cascade systems, see (4). The method only works for systems where both subsystems have order one. But this is in practice a common case.

The method consists of the following steps:

Step 1: Identify the first subsystem using data $u$ and $y_{1}$ with order $n_{1}$.

Step 2: Identify the extended observability matrix for the Single-Input Multiple-Output (SIMO) system from $u$ to $y_{1}$ and $y_{2}$ with order $n_{3}$. Denote the identified extended observability matrix

$$
O_{r} \tilde{T}=\left[\begin{array}{cc}
\xi_{1,1} & \xi_{1,2} \\
\eta_{1,1} & \eta_{1,2} \\
\vdots & \vdots \\
\xi_{r, 1} & \xi_{r, 2} \\
\eta_{r, 1} & \eta_{r, 2}
\end{array}\right]
$$

where $\xi_{i, 1}$ has size $\left(1 \times n_{1}\right)$ and $\eta_{i, 2}$ has size $\left(1 \times n_{3}-n_{1}\right)$. Step 3: From (4) it can be seen that for the first subsystem the state space matrices can be solved with least squares just as in the standard subspace formulation

$$
\begin{gathered}
\hat{C}_{1}=C_{1} \tilde{T}_{11}=\xi_{1,1} \\
\hat{A}_{1}=\arg \min _{A_{1}} \sum_{i=1}^{r-1}\left\|\xi_{i+1,1}-\xi_{i, 1} A_{1}\right\|_{2}^{2}
\end{gathered}
$$

Step 4: From (4) it is seen that for the second subsystem the estimate $\hat{C}_{2}$ is given by

$$
\hat{C}_{2}=C_{2} \tilde{T}_{22}=\eta_{1,2}
$$

In general it is not obvious how a a least square problem should be formulated for the second subsystem. To illustrate the concept we consider the special case when the order of the second subsystem is one. Denoting the matrix product $\chi=B_{2} C_{1}$ gives the following least square problem in $\hat{A}_{2}$ and $\chi$.

$$
\arg \min _{A_{2}, \chi} \sum_{i=1}^{r-1}\left\|\eta_{i+1,2}-\eta_{i, 1} A_{2}-\chi \xi_{i, 2}\right\|_{2}^{2}
$$

Step 5: When $\chi=B_{2} C_{1}$ has been found, $\hat{B}_{2}$ can be solved. Finally $\hat{B}_{1}$ can be calculated as in the standard subspace formulation as a linear regression problem.

\section{EXAMPLES}

In this section the two methods presented in this paper will first be applied to a real application, the double tank process, and then the first direct method will be applied to a simulated system to show how the method performs for higher order systems.

\section{A. The Double Tank Process}

The double tank system from Quanser Inc consists of two equivalent water tanks. A DC-motor drives a pump which pumps water from the basin into the upper tank. Water then flows out from the upper tank into the lower tank through a small outlet. The water from the lower tank then flows out into the basin. The input to the system is the input voltage to the DC-motor, $u$ and the outputs are the water level in each tank, $h_{1}$ and $h_{2}$.

The process is nonlinear. The outflow from one tank is proportional to the square root of the water level. The identification of the system will hence be performed around a linearization point.

\section{B. Identification}

A white Gaussian noise process is used as input during the identification. The sampling time is chosen as $T_{s}=1 \mathrm{~s}$. 300 samples of the input and outputs were collected, 200 used for identification and 100 used for validation. When all data has been collected, the identification process starts. The methods presented in section IV are used and their results are compared. First Method 1 is considered.

1) Method 1: The state space matrices, $\hat{A}_{1}, \hat{B}_{1}, \hat{C}_{1}, \hat{A}_{3}$ and $\hat{C}_{3}$ are identified with N4SID [1], [2]. The orders of these systems are chosen by looking at the singular values of their respective extended observability matrices.

The order of $G_{1}$ is chosen as $n_{1}=1$ and the order for $G_{3}$ is chosen as $n_{3}=2$.

A similarity transform is found by solving the optimization problem (8) numerically. Since the orders of the subsystems are both one, the rank condition is automatically fulfilled. When the transformation matrix $T$ and $\tilde{B}_{1}$ are found then it is straightforward to find $\hat{A}_{2}, \hat{B}_{2}$ and $\hat{C}_{2}$.

2) Method 2: Again the N4SID is used, but now on the SIMO system to get an estimate of the extended observability matrix. The singular values of the extended observability matrix suggest that the order should be chosen as 2. From previous results we know that the first subsystem is approximated well by a first order system. Method 2 is now applied to the extended observability matrix and the state space matrices for the two subsystems are calculated.

3) Results: The methods previous applied to the two tank process are compared to a physical model [12] and the $H_{\infty}$ method (2), [9].

For the first subsystem the two proposed methods and the $H_{\infty}$-method gives similar result. This is obvious since all three methods are estimating the first subsystem in the same way. They also have about the same dynamics as the physical model and only a very small difference in gain. The difference in gain could originate from wear and tear in the real process as well as from the discretization and linearization in the physical model.

The bode plot for the second system for the two different methods are shown in Fig. 2. For the second subsystem the methods gives slightly different results. First we can note that the estimated system using the $H_{\infty}$-method is of order 3 as expected. The dynamics are about the same for the two proposed methods, i.e. the eigenvalues of system matrix 


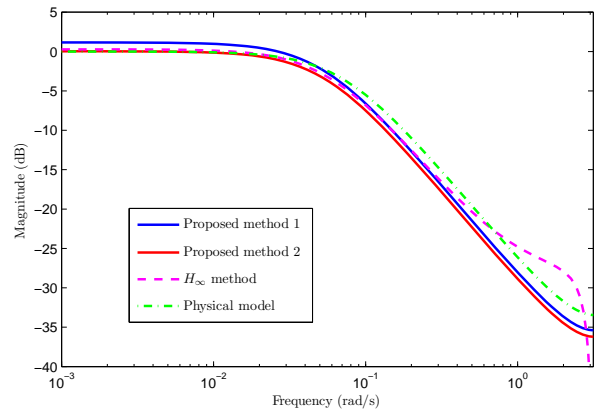

Fig. 2. Bode magnitude plot of the second subsystem $G_{2}$ for the two proposed method, the physical model and the $H_{\infty}$-method.

are close but they differ from the $H_{\infty}$-method. As the two tanks are equal we would expect the dynamics of the first and second subsystem to be equal. This can be seen in the physical model. This is the case in both of the proposed methods but not in the $H_{\infty}$-method.

The gain difference, that can be seen between the two proposed methods, is due to the different way the two methods calculates the state space matrices.

Finally the prediction error for the models given by the three methods, are calculated for the validation data, i.e.

$$
\begin{array}{r}
V\left(\hat{G}_{1}, \hat{G}_{2}\right)=\frac{1}{N} \sum_{t=1}^{N}\left(y_{1}(t)-\hat{G}_{1}(q) u(t)\right)^{2}+ \\
\left(y_{2}(t)-\hat{G}_{1}(q) \hat{G}_{2}(q) u(t)\right)^{2}
\end{array}
$$

The resulting prediction error is $4.2 \cdot 10^{-4}$ for the indirect method, $3.8 \cdot 10^{-4}$ for the direct method and $3.9 \cdot 10^{-4}$ for the $H_{\infty}$-method. The difference in prediction errors for the three methods is small.

It looks like the second method performs slightly better for this simple system. The execution time is also much shorter for this method since the optimization, i.e. the solving of the least square problem, is much more computational efficient than for the first method. But on the other hand, it is not obvious how the second method should be extended to handle larger systems.

\section{Higher order systems}

Here we consider a numerical example of a cascade system with higher order subsystems

$$
\begin{aligned}
& G_{1}(q)=\frac{q-0.1}{(q+0.6)(q-0.8)} \\
& G_{2}(q)=\frac{1}{\left(q^{2}-0.5 q+0.5\right)}
\end{aligned}
$$

The measurement noise variances are $\lambda_{1}=1$ and $\lambda_{2}=1$. The input is white Gaussian noise with unit variance. The system is simulated for 500 data points and the indirect method is then applied, this is repeated 100 times. The result of the Monte-Carlo simulation is shown for the second subsystem in Fig. 3. The first subsystem is the same as for direct application of the standard subspace method.

From the figure it can be seen that the method performs well for this system. It seems that the mean over the MonteCarlo simulations tends to the true system. The prediction

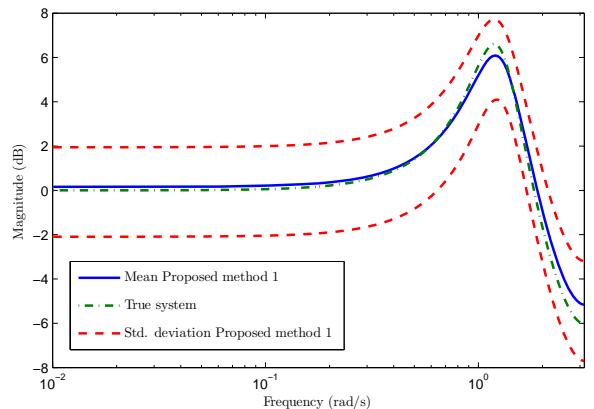

Fig. 3. The mean and standard deviation of the Monte-Carlo simulation for the second subsystem.

error (9) is comparable with the direct subspace method applied to the SIMO system from $u$ to $y_{1}$ and $y_{2}$.

\section{INPUT DESIGN FOR CASCADE SYSTEMS}

In the identification of the double tank system the input was chosen as a Gaussian white noise sequence. This it not generally the optimal input. The problem is that the input to the second system can not be chosen directly. We consider the input signal to the second subsystem

$$
u_{2}(t)=G_{1}(q) u(t)
$$

which consequently is directly colored by the the first unknown subsystem. Hence, a good input for identification of $G_{1}$ may give a bad input to $G_{2}$ and vice versa.

The recent framework [13] for experiment design, introduced by Hjalmarsson and co-workers could be interesting to apply to cascade systems. The main idea of this framework is that the estimation properties could be separated from the application specification.

To illustrate the framework an example is given for a simple cascade system. Consider the cascade system with first order FIR subsystems, i.e. $G_{1}(q)=1+b_{1} q^{-1}$ and $G_{2}(q)=1+b_{2} q^{-1}$. The model parameter vector is denoted $\theta=\left[\begin{array}{ll}b_{1} & b_{2}\end{array}\right]$ and assume that the true system can be described by $\theta_{0}=\left[\begin{array}{ll}b_{1}^{0} & b_{2}^{0}\end{array}\right]$.

Assume that we have some requirements on the parameter covariance, then the application specification could be written as

$$
V_{\text {app }}(\theta)=\left[\begin{array}{ll}
b_{1}-b_{1}^{0} & b_{2}-b_{2}^{0}
\end{array}\right] Q\left[\begin{array}{l}
b_{1}-b_{1}^{0} \\
b_{2}-b_{2}^{0}
\end{array}\right] \leq \frac{1}{\gamma}
$$

where $Q$ is some weighting matrix and $\gamma>0$ is some constant . This weighting matrix could for example be chosen such that the parameter in the second subsystem is more important than the parameter for the first subsystem or vice versa.

Now consider a typical system identification method that minimizes the mean square prediction error, i.e.

$$
\begin{aligned}
& \hat{\theta}_{N}=\arg \min _{\theta} V_{I D, N}(\theta) \\
& V_{I D, N}(\theta)=\frac{1}{N} \sum_{t=1}^{N} \frac{\left(y_{1}(t)-\left(1+b_{1} q^{-1}\right) u(t)\right)^{2}}{\lambda_{1}}+ \\
& \frac{1}{N} \sum_{t=1}^{N} \frac{\left(y_{2}(t)-\left(1+b_{1} q^{-1}\right)\left(1+b_{2} q^{-1}\right) u(t)\right)^{2}}{\lambda_{2}}
\end{aligned}
$$


We will now try to find the optimal input for this problem. The input should be optimal in the sense that it minimizes the input power such that the application specifications are fulfilled. This is known as least costly identification [14]. Using the framework [13], asymptotically $(N \rightarrow \infty)$ it can be shown that this optimization problem can be formulated as

$$
\begin{aligned}
\min _{u(t)} & E\left[u(t)^{2}\right] \\
\text { s.t } & \gamma V_{\text {app }}^{\prime \prime} \leq N V_{\text {ID }}^{\prime \prime}
\end{aligned}
$$

where the constraint is a matrix inequality.

For the simple system presented above the optimization problem becomes

$$
\begin{array}{cc}
\min _{r_{0}} & r_{0} \\
\text { s.t } \quad & N\left[\begin{array}{ll}
\rho_{1} & \rho_{2} \\
\rho_{2} & \rho_{3}
\end{array}\right] \geq \gamma Q \\
& r_{0} \geq r_{1} \\
\rho_{1}=r_{0} / \lambda_{1}+\left[\left(1+b_{2}^{2}\right) r_{0}+2 b_{2} r_{1}\right] / \lambda_{2} \\
\rho_{2}=\left[\left(1+b_{1}^{2}\right) r_{0}+2 b_{1} r_{1}\right] / \lambda_{2} \\
\rho_{3}=\left[\left(1+b_{1} b_{2}\right) r_{0}+\left(b_{1}+b_{2}\right) r_{1}\right] / \lambda_{2}
\end{array}
$$

where $r_{\tau}=\bar{E}[u(t) u(t-\tau)]$. The optimization problem is solved for $r_{0}$ and $r_{1}$ and a optimal input with this covariance function could be generated.

Let us look at some special cases. First assume that $\lambda_{2} \gg \lambda_{1}$ and that the parameter $b_{2}$ is unimportant. Then we get $r_{1}=0$, i.e. the input should be chosen as white noise as expected, we know that this is a optimal input for the MA(1) process. If we instead consider $\lambda_{1} \gg \lambda_{2}$ and that the first parameter, $b_{1}$ is unimportant, then we would want a white noise input to the second subsystem. If the optimization problem above is solved the input becomes a white noise sequence filtered through the inverse of the first subsystem, hence the input to the second becomes white noise.

In general the input becomes an optimal weighting between these two cases depending on the noise levels and which parameter that is more important.

For more complex systems, the problem above cannot be solved for the covariance function $r_{\tau}$ but the optimization problem must be solved for a parametrization of the spectrum of $u(t)$, see [13]. It would be interesting to study the optimal input for more complex systems.

\section{CONCLUSIONS}

The problem considered in this paper has been inspired by the discussions on the industrial applications of structural system identification given in [3]. Two new methods for identification of cascade systems has been presented. Both methods show promising results, their performances are comparable to state of the art methods for estimating cascade structured systems. But a more thorough analysis has to be done to verify the performance.

The first method involves solving a nonlinear optimization problem. This optimization should be further studied. The problem is solved with a numerical method and there could be problems with local minima points. The second method on the other hand is computationally less costly but it is not obvious how it can be extended to systems with order larger than one. It would be interesting to study, if it is even possible, how this method could be extended to larger systems.

The statistical properties of the model estimates are hard to analyze. But the methods could be used to find initial estimates to Prediction error methods or Maximum likelihood methods, this would be interesting to explore further.

The problem of optimal input design for cascade systems was briefly reviewed. This framework should be analyzed for more complex systems and it would be interesting to study other applications specification, for example requirements on the model given by feedback specifications.

\section{REFERENCES}

[1] L. Ljung, System Identification: Theory for the User, 2nd Edition. Upper Saddle River, New Jersey: Prentice Hall, 1999.

[2] P. van Overschee and B. DeMoor, Subspace Identification of Linear Systems: Theory, Implementation, Applications. Kluwer, 1996.

[3] B. Wahlberg, M. Jansson, T. Matsko, and M. Molander, "Experiences from subspace system identification - comments from process industry users and researchers," in Modeling, Estimation and Control, Lecture Notes in Information and Control Sciences. Springer, Oct. 2007, pp. 315-327.

[4] B. Wahlberg, H. Hjalmarsson, and J. Mårtensson, "Variance analysis for identification of cascade systems," in Decision and Control, 2008. CDC 2008. 47th IEEE Conference on, Dec. 2008, pp. 131-136.

[5] B. Wahlberg, H. Hjalmarsson, and J. Mårtensson, "Variance results for identification of cascade systems," Automatica, vol. 45, pp. 14431448, Jun. 2009.

[6] C. Lyzell, M. Enqvist, and L. Ljung, "Handling certain structure information in subspace identification," in Proceedings of the 15th IFAC Symposium on System Identification, Apr. 2009.

[7] T. Van Gestel, J. Suykens, P. Van Dooren, and B. De Moor, "Identification of stable models in subspace identification by using regularization," Automatic Control, IEEE Transactions on, vol. 46, no. 9, pp. $1416-1420$, sep 2001.

[8] S. Lacy and D. Bernstein, "Subspace identification with guaranteed stability using constrained optimization," Automatic Control, IEEE Transactions on, vol. 48, no. 7, pp. 1259 - 1263, july 2003.

[9] B. Wahlberg and H. Sandberg, "Cascade structural model approximation of identified state space model," in Proceedings IEEE Conference on Decision and Control, Cancun, Mexico, Dec. 2008.

[10] B. Wahlberg, H. Hjalmarsson, and J. Mårtensson, "On identification of cascade systems," in 17th IFAC World Congress, Jul. 2008, pp. 5036-5040.

[11] R. A. Horn and C. R. Johnson, Matrix Analysis. Cambridge University Press, 1990.

[12] K. Johansson, A. Horch, O. Wijk, and A. Hansson, "Teaching multivariable control using the quadruple-tank process," Decision and Control, 1999. Proceedings of the 38th IEEE Conference on, vol. 1, pp. $807-812$ vol.1, 1999.

[13] H. Hjalmarsson, "System identification of complex and structured systems," in European Control Conference, Budapest, Hungary, Aug. 2009, pp. 3424-3452.

[14] X. Bombois, G. Scorletti, M. Gevers, P. V. den Hof, and R. Hildebrand, "Least costly identification experiment for control," Automatica, vol. 42, no. 10, pp. 1651 - 1662, 2006. [Online]. Available: http://www.sciencedirect.com/science/ article/B6V21-4KR85M7-1/2/23969a3b1ef2e293b63428b4d3fc62a7 